\title{
Stabilizing open quantum batteries by sequential measurements
}

\author{
Stefano Gherardini $\odot,{ }^{1,}{ }^{*}$ Francesco Campaioli, ${ }^{2}$ Filippo Caruso, ${ }^{1}$ and Felix C. Binder $\odot^{3}$ \\ ${ }^{1}$ Department of Physics and Astronomy \& LENS, University of Florence, via G. Sansone 1, I-50019 Sesto Fiorentino, Italy \\ ${ }^{2}$ School of Physics and Astronomy, Monash University, Victoria 3800, Australia \\ ${ }^{3}$ Institute for Quantum Optics and Quantum Information - IQOQI Vienna, Austrian Academy of Sciences, \\ Boltzmanngasse 3, A-1090 Vienna, Austria
}

(Received 10 October 2019; published 29 January 2020)

\begin{abstract}
A quantum battery is a work reservoir that stores energy in quantum degrees of freedom. When immersed in an environment, an open quantum battery needs to be stabilized against free-energy leakage due to decoherence, unavoidably entailing entropy production. For this purpose we here propose a stabilization protocol given by a nonunitary open-loop control action able to compensate for the entropy increase and to maintain the open quantum battery in its highest ergotropy state. The protocol relies on nonselective, frequent, projective measurements that are interspersed by optimized time intervals. In accordance with a second-law-like inequality derived for the entropy production rate of the controlled battery, the proposed procedure results in minimized control power. The effectiveness of the method is finally tested on a qubit subject to decoherence, achieving an average fidelity value around $95 \%$.
\end{abstract}

DOI: 10.1103/PhysRevResearch.2.013095

\section{INTRODUCTION}

Among recent research in quantum thermodynamics [1-5], the design of quantum energy storage devices, called quantum batteries [6-20], is of increasing interest. So far the main focus has been on multipartite speed-up effects in charging [7,9-12,15-17], fluctuations in charging precision $[8,13,17,19]$, and mitigating imprecise unitary control pulses [20]. A specific quantum battery design using decoherencefree subspaces has been recently proposed as to avoid energy leakage into the environment [21]. However, no attention has been paid to the energetics of efficiently stabilizing quantum batteries that do not benefit from such protection-affording symmetries, even if contributions in the area of control theory [22,23] touch upon this question both in classical [24] and in quantum settings [25-28].

In this paper, we introduce the concept of an open quantum battery (OQB). Here, the quantum system $\mathcal{B}$, acting as a battery, interacts with the surrounding environment $\mathcal{E}$ leading to decoherence [29]. Due to this interaction, the entropy of the battery increases [30-33] and thus unitary control pulses applied to the system are not generally sufficient to compensate such entropy production and then stabilize the system; rather, we would require a source of free energy, such as a low-temperature heat bath. For this purpose, we propose a stabilization scheme based on a sequence of repeated quantum measurements $[34,35]$, each of them preserving the trace of

\footnotetext{
*gherardini@lens.unifi.it

Published by the American Physical Society under the terms of the Creative Commons Attribution 4.0 International license. Further distribution of this work must maintain attribution to the author(s) and the published article's title, journal citation, and DOI.
}

the system, i.e., no postselection is performed, as shown in Refs. [36-38]. The adoption of quantum measurements has been recently proposed for the realization of a quantum Maxwell's demon engine [39] and in Ref. [40] to fuel a cooling engine. Our goal is to neutralize the local increase of entropy and ensure energy-efficient control operations for implementing fast, on-demand charging/discharging and stabilization protocols, using the lowest amount of energy.

\section{OPEN QUANTUM BATTERIES}

A quantum battery is a finite-dimensional quantum system $\mathcal{B}$ whose energy is quantified by a bounded internal Hamiltonian $H_{0}$. We here consider the highest-energy state $|e\rangle$ and the lowest-energy state $|g\rangle$, both eigenstates of $H_{0}$, representing the maximally charged and discharged battery states, respectively. Hence, the battery's capacity [10] is simply given by $E_{\max } \equiv \operatorname{Tr}\left[H_{0}\left(\rho_{e}-\rho_{g}\right)\right]$, with $\rho_{e} \equiv|e\rangle\langle e|$ and $\rho_{g} \equiv|g\rangle\langle g|$. If the battery system were perfectly isolated it would always evolve unitarily. In contrast, an open quantum battery, when left uncontrolled, evolves under the effect of some open dynamics, i.e., $\dot{\rho}_{t}=-i\left[H_{0}, \rho_{t}\right]+\mathcal{D}\left[\rho_{t}\right]$, where, here and below, $\hbar$ is set to $1, \rho_{t}$ denotes the density operator of the system at time $t$, and $\mathcal{D}$ is the superoperator modeling free-energy leakage due to decoherence. Equivalently, the evolution of the system can also be described by means of a time-parametrized family of completely positive and trace-preserving (CPTP) maps $\Lambda_{t}: \rho_{0} \rightarrow \rho_{t}$, where $\rho_{t}=\Lambda_{t}\left[\rho_{0}\right]$, with steady state $\bar{\rho} \equiv$ $\lim _{t \rightarrow \infty} \Lambda_{t}\left[\rho_{0}\right]$ and initial density operator $\rho_{0}$.

A charging protocol must be able to powerfully charge the battery, bringing it into the excited state $|e\rangle$ from an arbitrary initial condition, e.g., a thermal state at inverse temperature $\beta$ or a state in the neighborhood of $|g\rangle$, so as to maximize its ergotropy [41], i.e., the amount of energy that can then be unitarily extracted. While a closed quantum 


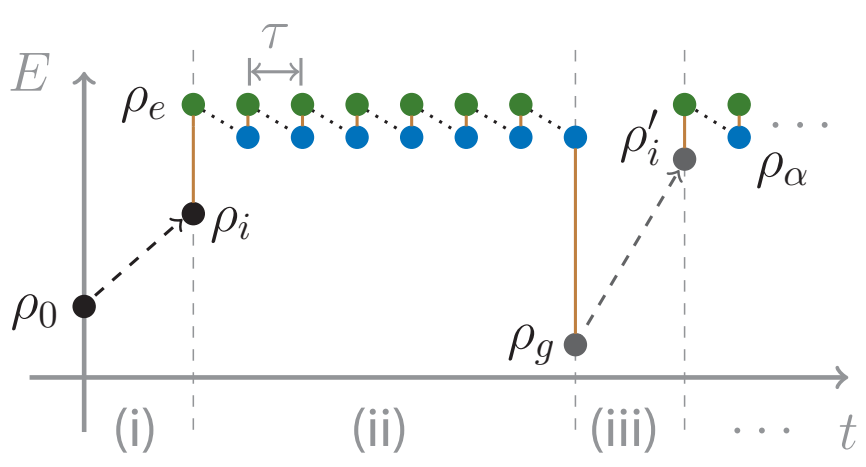

FIG. 1. Stabilization scheme-single-run illustration. After the initialization step (with initial state $\rho_{0}$ ), the battery stabilization protocol consists of intermittent free evolutions and fast unitary controlled dynamics [dotted points, corresponding to steps (i) and (iii) of the procedure] and projective measurements [solid brown line, step (ii)] in time intervals of duration $\tau$. In particular, the green dots denote the maximum energy state $\rho_{e}$, while the blue dots represent the state $\rho_{\alpha}$ of the battery immediately before a projective measurement in the Zeno regime. $\rho_{i}$ and $\rho_{i}^{\prime}$ are the nearest states to $\rho_{e}$ on the unitary orbit of $\rho_{0}$ and $\rho_{g}$, respectively.

battery can be charged by means of cyclic unitary operations, an open quantum battery experiences nonequilibrium freeenergy leakage. Hence, a unitary process no longer suffices to restore the battery state or to avoid the energy losses during its dynamics. Moreover, the target state $\rho_{e}$ does not generally belong to the unitary controllability space [23] of the system, especially if the charging time $t_{c}$ is comparable with $1 / \gamma$, where $\gamma$ denotes the relevant decoherence coefficient of the superoperator $\mathcal{D}$. So, how can an open quantum battery be charged and stabilized?

\section{STABILIZATION SCHEME}

In this paper, we propose a nonunitary (NU) control protocol that counteracts the increase of entropy induced by the interaction with the environment. The control scheme is realized by a sequence of projective measurements and intermittent driving as illustrated in Fig. 1. The first objective is to charge the battery by bringing it towards the excited state $\rho_{e}$. The second one is to maintain the system in the neighborhood of $\rho_{e}$ during the time interval $\left[0, t_{\text {fin }}\right]$. Now, we introduce each step of the stabilization protocol.

(i) Initialization. Given an input state $\rho_{0}$, the battery $\mathcal{B}$ is driven to that out-of-equilibrium state $\rho_{i}$ on its control orbit which lies closest to $\rho_{e}$ [e.g., in terms of the trace distance $\left.T(\rho, \sigma) \equiv \frac{1}{2} \operatorname{Tr}\left[\sqrt{(\rho-\sigma)^{2}}\right]\right]$. In general, this operation combines the uncontrollable open system dynamics with Hamiltonian control $H(t)=H_{0}+V(t)$. While magnitude constraints on the driving may impose bounds on the maximum achievable power [11], we here assume that driving fields of arbitrary magnitude are permitted. This allows for this step of the protocol to be performed sufficiently fast so as to be considered unitary, despite the presence of decoherence. However, also regimes of slow control could be equally treated by properly adapting existing control methods to the present scenario [42]. (ii) Quantum measurements and Zeno protection. After driving $\mathcal{B}$ into the state $\rho_{i}$, a projective energy measurement (in the eigenbasis of $H_{0}$ ) is performed on the battery: With probability $P_{e} \equiv \operatorname{Tr}\left[\rho_{i} \rho_{e}\right]$ the state of $\mathcal{B}$ collapses into the excited state, while with probability $P_{g} \equiv 1-P_{e}$ the collapse occurs into one of the other energy eigenstates. After the measurement, if $\mathcal{B}$ has collapsed into the maximum energy state $\rho_{e}$, then a Zeno protection protocol is applied. The latter consists of a sequence of frequent projective measurements (again in the energy eigenbasis) at discrete periodic times with the aim of freezing the dynamics of the battery and thus stabilizing it in the excited state. As proved in Refs. [43-45], the time interval $\tau$ between two consecutive measurements has to be chosen according to the relation $\Delta^{2} H_{\text {Zeno }} \tau^{2} \ll 1$, where $\Delta^{2} H_{\text {Zeno }}$ is the variance of the effective Zeno Hamiltonian $H_{\text {Zeno }} \equiv \rho_{e} H_{0} \rho_{e}=E_{e} \rho_{e}\left(E_{e} \equiv \operatorname{Tr}\left[H_{0} \rho_{e}\right]\right)$ with respect to the freezing state. This physically means that the battery is repeatedly brought back to the maximum energy state $\rho_{e}$ with a probability almost equal to one as long as $\rho_{e}$-the state to be stabilized-and the quantum state after the evolution are statistically indistinguishable, i.e., their difference is nondetectable by any measurement device [46]. Thus, for an experimental realization of the protocol, $\tau$ needs to be significantly smaller than both the timescale of the system dynamics and the characteristic decoherence time.

(iii) Reinitialization. If the projective energy measurement results in one of the lower-energy eigenstates, the stabilization procedure is repeated from the beginning, and unitary driving is applied as in step (i). This means that the whole procedure is repeated until the Zeno protection protocol starts.

To summarize, $\rho_{e}$ is an unstable state of the battery due to interactions with the environment. Hence, in order to stabilize $\mathcal{B}$, we apply the nonunitary process given by a sequence of projective measurements. Despite the probabilistic nature of this scheme, a very high fidelity $\mathcal{F} \equiv\left(\operatorname{Tr} \sqrt{\sqrt{\rho_{e}} \rho_{t} \sqrt{\rho_{e}}}\right)^{2}$ in stabilizing the battery can be achieved, as shown in Fig. 2 for an example process. Further details on numerical simulations are provided in Appendix B.

\section{Performance measures}

For each time $t$ we define two figures of merit for the stabilization scheme. First, the ratio

$$
\varsigma_{\mathrm{stab}}(t) \equiv \frac{\left\langle W_{\mathrm{stab}}(t)\right\rangle}{E_{\max }}
$$

is named the relative stabilization cost, where $\langle\cdot\rangle$ denotes the average over a sufficiently large number of protocol realizations and $W_{\text {stab }}(t)$ is the energy expended to stabilize $\mathcal{B}$. Second, we want to identify the excess cost of the stabilization procedure besides the energy cost spent to just compensate decoherence. To this end, we introduce the relative excess stabilization cost, i.e.,

$$
\xi_{\text {stab }}(t) \equiv \frac{\left|\left\langle W_{\text {stab }}(t)\right\rangle-\langle\Delta L(t)\rangle\right|}{E_{\max }},
$$

where $\langle\Delta L(t)\rangle$ is the average energy leakage that would spontaneously occur if the battery were left uncontrolled. Note that $\zeta_{\mathrm{stab}}(t)$ is a cumulative energy term: It is zero when no control is applied, but can also diverge since $\left\langle W_{\text {stab }}(t)\right\rangle$ is an 

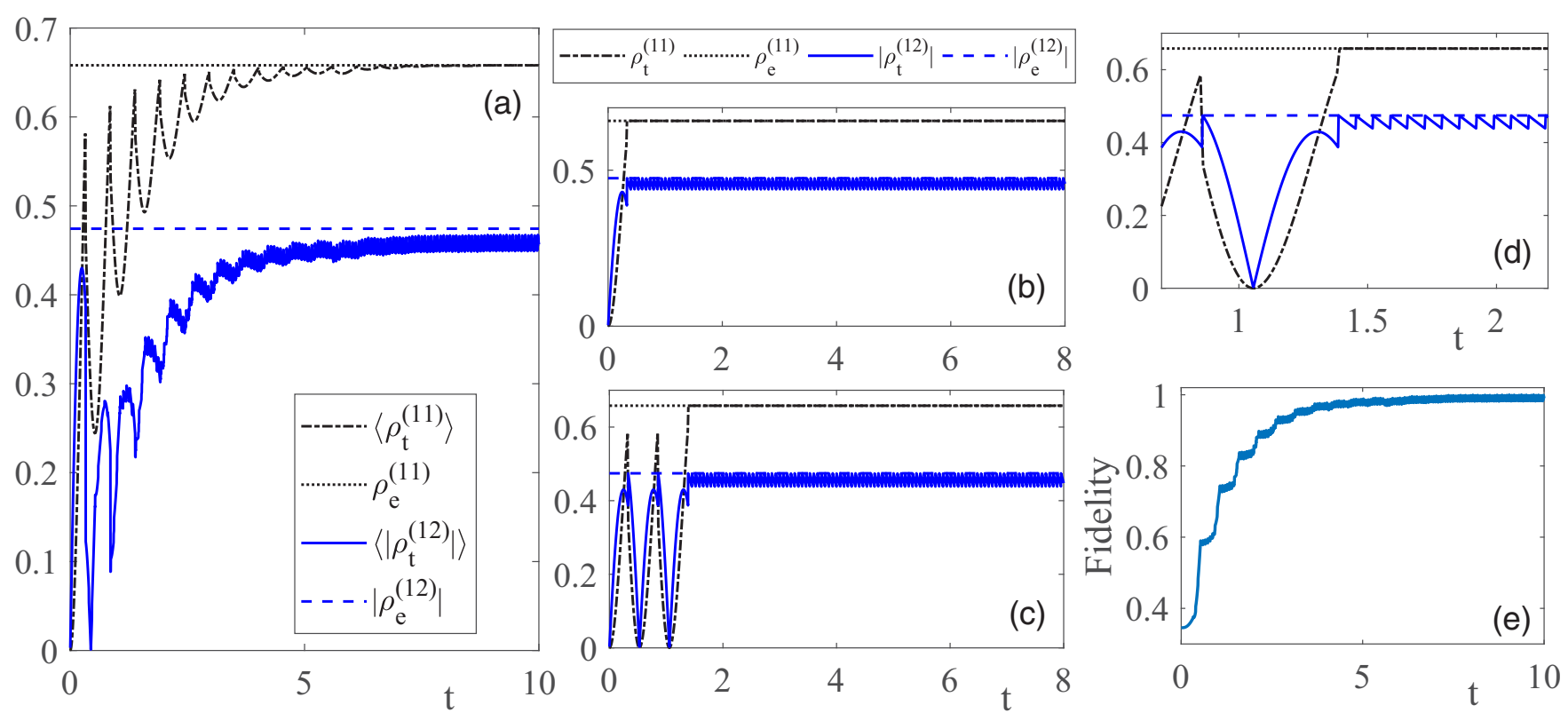

FIG. 2. Stabilization scheme-numerical results for a qubit with internal Hamiltonian $H_{0}=3 \sigma_{x}+\sigma_{z}$ (in natural units). (a) Average behavior over time of the battery density matrix, obtained by repeating the stabilization procedure 1000 times. (b), (c) Behavior over time of the battery density matrix in single realizations of the scheme: Being probabilistic, the charging process could require the application of more than one projective measurement. In the subplots, $\left(\rho_{e}^{(11)}, \rho_{e}^{(12)}\right)$ and $\left(\rho_{t}^{(11)}, \rho_{t}^{(12)}\right)$ are the top diagonal elements and the coherence terms, respectively, of the maximum energy state $\rho_{e}$ of the qubit battery and of the corresponding time-evolved density matrix $\rho_{t}$. (d) Zoom of (c) in the time interval $[0.75,2.15]$, showing the occurrence of a failure collapse and the resulting reinitialization procedure. Further details can be found in Appendix B. (e) Stabilization fidelity $\mathcal{F}$ over 1000 realizations of the stabilization procedure. At $t=0, \mathcal{F}$ starts from a value in the range $[0.3,0.4]$ since also the initialization step has been taken into account.

unbounded quantity. Thus, it can be easily adapted for the definition of the relative stabilization rate

$$
R_{\text {stab }} \equiv \lim _{t \rightarrow \infty} \frac{\zeta_{\text {stab }}(t)}{t},
$$

which would be the same if defined in terms of $\xi_{\text {stab }}(t)$ rather than $\zeta_{\text {stab }}(t)$, due to the long term limit. This leads us to just consider the stabilization power $\mathfrak{P}_{\text {stab }}=\dot{W}_{\text {stab }}$ as a performance measure in the following section.

\section{MINIMUM CONTROL POWER}

Here, we prove a bound providing the minimum power required to stabilize the OQB. It originates from a secondlaw-like inequality for the battery's irreversible entropy production rate $\Sigma\left(\rho_{t}\right)$ (see Appendix A for the proof). A similar result can be found in Ref. [26], but concerning the energy cost to coherently control a mesoscopic quantum system. In our open-loop control framework, the entropy production rate $\Sigma\left(\rho_{t}\right)$ equals the sum of two contributions, $\Sigma_{D}\left(\rho_{t}\right)$ and $\Sigma_{\mathrm{NU}}\left(\rho_{t}\right)$, denoting respectively the entropy production rates due to environmental decoherence and the effect of the observer/experimenter, responsible for the nonunitary control of the battery. In particular, as discussed in Appendix A, the entropic contribution $\Sigma_{\mathrm{NU}}\left(\rho_{t}\right)$ is equal to the time derivative of the Shannon entropy $H(P) \equiv-\sum_{k \in\{e, g\}} P_{k} \log P_{k}$, i.e.,

$$
\Sigma_{\mathrm{NU}}\left(\rho_{t}\right)=\dot{H}(P)=-\dot{P}_{e} \log \left(\frac{P_{e}}{1-P_{e}}\right),
$$

with $P_{k}$ 's probabilities that the battery collapses in one of the energy eigenstates. Indeed, to each of those probabilities is associated the information content of the measurement outcomes, which are stored in a classical memory [47]. This means that, while the measurement procedure locally reduces the battery's entropy, the reading and storing of the measurement outcomes entails an additional entropy production which cannot be neglected. According to Landauer's principle [48-50], the irreversible erasure of such information leads to an energy consumption, proportional to the temperature of the thermal bath used in the erasure procedure.

Once again, it is worth noting that, since $\mathcal{B}$ is affected by decoherence, the evolution of the battery admits at least one fixed point denoted as $\bar{\rho}_{\mathrm{dec}}$. For the case of a qubit, the steady state $\bar{\rho}_{\text {dec }}$ (with no coherence in the energy eigenbasis) can always be described by an effective temperature $T_{\bar{\rho}_{\text {dec }}}$. The latter is interpreted as the physical temperature of a fictitious quantum system that would lead to the same decoherence effects. In particular, as shown in Appendix A, by defining $E\left(\rho_{t}\right)$ and $E_{D}\left(\rho_{t}\right)$, respectively, as the battery's total energy and the energy driven into $\mathcal{B}$ by the environment, the control power $\dot{W}_{\text {stab }}$ obeys the following inequality,

$$
\mathfrak{P}_{\mathrm{stab}} \equiv \dot{W}_{\mathrm{stab}}\left(\rho_{t}\right) \geqslant \dot{E}\left(\rho_{t}\right)-T_{\overline{\bar{\rho}}_{\mathrm{dec}}} \dot{S}_{D}\left(\rho_{t}\right),
$$

with $S_{D}$ denoting the von Neumann entropy of the uncontrolled battery (note that $\dot{S}_{D} \propto \Sigma_{D}$ ). The lower bound (5) can be recast into the inequality

$$
\dot{F}\left(\rho_{t}\right) \leqslant 0, \quad \text { with } \quad F \equiv E_{D}-T_{\bar{\rho}_{\text {dec }}} S_{D}
$$


the battery free energy. This inequality represents the second law of thermodynamics: The free energy of the uncontrolled battery reduces due to the increase of the von Neumann entropy $S_{D}$ resulting from the open systems dynamics. Therefore, the minimum value of $\dot{W}_{\text {stab }}$ implies the equality $\dot{F}\left(\rho_{t}\right)=$ 0 , with the result that the lowest energy $W_{\text {stab }}^{(\min )}$ required to control $\mathcal{B}$ (note that, apart from a constant term, $W_{\text {stab }}^{(\min )}$ is equal to $E-T_{\bar{\rho}_{\mathrm{dec}}} S_{D}$ ) is such that the free energy is constant, i.e., the increase in entropy due to the environment is compensated by the control operation. Note that Eq. (5) is valid regardless of the control action applied on $\mathcal{B}$, and for this reason the symbol $\langle\cdot\rangle$ has not been used. However, for the probabilistic stabilization procedure we are proposing, the results from Eq. (5) just hold true only on average.

\section{ENERGETIC EFFICIENCY}

Returning to Eq. (2), we now derive the average control energy $\left\langle W_{\text {stab }}(t)\right\rangle$ and environmental losses $\langle\Delta L(t)\rangle$ for an open quantum battery controlled by a sequence of projective measurements. In particular, the energetic balance equation of the OQB is characterized within the total time interval $\left[0, t_{\max }\right]$ and evaluated in the limit of fast control, i.e., $V(t)=0$ almost for any $t$.

The battery's energy is determined by the timeindependent Hamiltonian $H_{0}$. Thus, the cost for the initialization of the battery and its dynamical evolution is exactly equal to

$$
\Delta E_{\text {evol }}=\operatorname{Tr}\left[H_{0}\left(\rho_{i}-\rho_{0}\right)\right] .
$$

Indeed, for fast control, the integral $\int \operatorname{Tr}\left[V(t)\left(\rho_{t}-\rho_{0}\right)\right] d t$ is negligible and $\int \rho_{t} d t \approx \rho_{i}$. On the other hand, the cost $\Delta E_{\text {meas }}$ of each projective measurement is given by the difference between the battery energies, respectively, after and before the measurement,

$$
\begin{aligned}
& \Delta E_{\text {meas }} \\
& \quad= \begin{cases}\operatorname{Tr}\left[H_{0}\left(\rho_{e}-\rho_{i}\right)\right], & \text { with probability } P_{e}=\operatorname{Tr}\left[\rho_{i} \rho_{e}\right], \\
\operatorname{Tr}\left[H_{0}\left(\rho_{i}-\rho_{g}\right)\right], & \text { with probability } P_{g}=1-P_{e} .\end{cases}
\end{aligned}
$$

However, on average there is no energetic cost associated with the measurement, i.e., $\left\langle\Delta E_{\text {meas }}\right\rangle=0$, independently of $\rho_{i}$. Only the entropic cost for the erasure of the measurement information has to be considered. The latter is equal to $\beta^{-1} H(P)$, where $\beta$ denotes the inverse temperature associated with the thermal reservoir allowing for the resetting of the memory after each measurement. The same holds true for the Zeno protection protocol, whereby the energy cost of a projection on the energy basis is equal on average to $\beta^{-1} H\left(P\left(\rho_{\alpha}\right)\right)$, such that overall one has that

$$
\left\langle\Delta E_{\text {Zeno }}\right\rangle=\bar{m} \beta^{-1} H\left(P\left(\rho_{\alpha}\right)\right),
$$

where $\bar{m}$ denotes the average number of projections in the Zeno regime (Zeno measurements) [51] and $\rho_{\alpha}$ is the average state of the battery immediately before each Zeno measurement, depending on the measurement frequency $1 / \tau$. It is worth noting that also during the Zeno protection procedure the average measurement cost $P_{e}\left(\rho_{\alpha}\right) \operatorname{Tr}\left[H_{0}\left(\rho_{e}-\rho_{\alpha}\right)\right]+$ $P_{g}\left(\rho_{\alpha}\right) \operatorname{Tr}\left[H_{0}\left(\rho_{g}-\rho_{\alpha}\right)\right]$ is a vanishing quantity. As a result, since the stabilization procedure is repeated with probability $P_{e}$ until the battery is charged, i.e., $\rho_{t}$ reaches $\rho_{e}$, the average total work needed to keep the energy storage in the battery until $t=t_{\text {fin }}$ is given by the following relation,

$$
\left\langle W_{\text {stab }}\left(t_{\text {fin }}\right)\right\rangle \approx\left(1+\sum_{k=1}^{\bar{N}} P_{g}^{k}\right) \Delta E_{\text {evol }}+\left\langle\Delta E_{\text {Zeno }}\right\rangle,
$$

where $\bar{N}$ is the average number of times the stabilization procedure is repeated with probability $P_{g}$.

Then, let us derive the average total energy leakages $\left\langle\Delta L\left(t_{\text {fin }}\right)\right\rangle$. Since the battery is an open quantum system, its evolution entails energy leakages, which are equal to

$$
\Delta L_{\mathrm{evol}}=\int \operatorname{Tr}\left[H_{0} \mathcal{D}\left[\rho_{t}\right]\right] d t,
$$

the integrated energy flow between $\mathcal{B}$ and the environment. In contrast, the projective measurements, assumed as discontinuous operations, are not affected by the environment. This assumption is clearly just an abstraction, which may be extended to nonideal measurements with an inherent energy consumption [52]. During the Zeno protection procedure, losses are on average equal to

$$
\left\langle\Delta L_{\text {Zeno }}\right\rangle=\sum_{k} \int_{t_{k}}^{t_{k}+\tau} \operatorname{Tr}\left[H_{0} D\left(\rho_{t}\right)\right] d t,
$$

where $\tau$ is the time interval between two consecutive Zeno measurements. In conclusion, this implies that the average total energy leakage $\left\langle\Delta L\left(t_{\text {fin }}\right)\right\rangle$ at the final time instant $t_{\text {fin }}$ is

$$
\left\langle\Delta L\left(t_{\mathrm{fin}}\right)\right\rangle \approx\left(1+\sum_{k=1}^{\bar{N}} P_{g}^{k}\right) \Delta L_{\mathrm{evol}}+\left\langle\Delta L_{\mathrm{Zeno}}\right\rangle,
$$

with $\Delta L_{\text {evol }}$ counted $\bar{N}$ times until $\rho_{t}=\rho_{e}$.

The minimal requirement for the battery stabilization is the equality between the average total work $\left\langle W_{\text {stab }}\left(t_{\max }\right)\right\rangle$ and the accumulated losses until the time instant $t_{\max }$ denoted as break-even time. An upper bound of the break-even time can be computed just by inverting the relation $\left\langle W_{\text {stab }}\left(t_{\max }\right)\right\rangle=$ $E_{\max }$. However, to make $\mathcal{B}$ a high-performance battery, the collapse probability $P_{g}$ has to be as close as possible to zero in the shortest time interval, and this is in contrast with the need for low-energy consumption. Thus, during the initialization step of the procedure, a trade-off between precision and energy cost is unavoidable. Similar results are also observed during the Zeno protection protocol. In this regard, let us consider the average stabilization power $\left\langle\mathfrak{P}_{\text {stab }}\right\rangle=\left\langle\dot{W}_{\text {stab }}\right\rangle \approx$ $\left\langle W_{\text {stab }}(\tau)\right\rangle / \tau$. By relating Eqs. (10) and (13), one determines that $\left\langle\mathfrak{P}_{\mathrm{stab}}(\tau)\right\rangle$ is approximately equal to

$$
\left\langle\mathfrak{P}_{\text {stab }}(\tau)\right\rangle \approx\left[\operatorname{Tr}\left[H_{0}\left(\rho_{i}-\rho_{0}\right)\right]+\bar{m} \beta^{-1} H\left(P\left(\rho_{\alpha}\right)\right)\right] / \tau,
$$

where the first and second terms of Eq. (14) denote, respectively, the average cost per cycle to initially bring the battery close to $\rho_{e}$, and the Landauer cost per cycle to reset the (classical) memory register. We can thus conclude that the longer is $\tau$, the smaller is the value of the power required to stabilize on average the battery, but the less accurate will be the precision to bring it on $\rho_{e}$. 
As a final remark, it is worth observing that for a vanishing value of $P_{g}$ we can perform a first-order expansion of both $\left\langle W_{\text {stab }}\left(t_{\text {fin }}\right)\right\rangle$ and $\left\langle\Delta L\left(t_{\text {fin }}\right)\right\rangle$ as a function of $P_{g}$, so that $\left\langle W_{\text {stab }}\left(t_{\text {fin }}\right)\right\rangle \approx\left(1+P_{g}\right) \Delta E_{\text {evol }}+\left\langle\Delta E_{\text {Zeno }}\right\rangle$ and $\left\langle\Delta L\left(t_{\max }\right)\right\rangle \approx$ $\left(1+P_{g}\right) \Delta L_{\text {evol }}+\left\langle\Delta L_{\text {Zeno }}\right\rangle$. Therefore, if we also reasonably assume that in the Zeno regime the sum of the energy losses is on average almost equal to the energy required to charge the quantum system (i.e., $\left\langle\Delta E_{\text {Zeno }}\right\rangle \approx\left\langle\Delta L_{\text {Zeno }}\right\rangle$ ), the relative excess stabilization cost at $t=t_{\mathrm{fin}}$ is given by the following relation,

$$
\xi_{\text {stab }}\left(t_{\text {fin }}\right) \approx \frac{1+P_{g}}{E_{\max }}\left|\Delta E_{\text {evol }}-\Delta L_{\text {evol }}\right|,
$$

with the result that $\xi_{\text {stab }}\left(t_{\text {fin }}\right)=0$ if the energy cost to drive the battery up to the state $\rho_{i}$ perfectly equals the decoherence losses during the battery evolution (i.e., if the losses are just compensated by the control action).

\section{Entropic cost in the Zeno regime}

Now, let us analyze in more detail the entropic cost of the Zeno protection procedure, based on applying a sequence of projective energy measurements. In this regard, at the level of the battery, the shorter is $\tau$, the closer the density operator of $\mathcal{B}$ approaches $\rho_{e}$. However, such a condition does not imply that the global variation of energy during the procedure decreases in the same way. Indeed, the smaller is $\tau$, the greater is the average number of Zeno measurements, each of them entailing an entropic cost proportional to the irreversible loss of the information content. From a purely dynamical point of view, this corresponds to the cost of purifying the state of the open quantum battery in correspondence to $\rho_{e}$. As a result, an increasing of the Landauer cost, corresponding to a more frequent memory erasure, is expected. Such behavior is illustrated by Fig. 3, in which the entropy production

$$
\sigma_{\text {Zeno }} \equiv m \int_{0}^{\tau} \Sigma_{\mathrm{NU}}\left(\rho_{t}\right) d t=m \int_{0}^{\tau} \dot{H}(P) d t
$$

is plotted as a function of $\tau$ by fixing the duration $T_{\text {Zeno }}$ of the procedure. In Fig. 3, the Landauer entropic cost, being proportional to $1 / \tau$, diverges as $\tau$ decreases to zero, and the behavior of $\sigma_{\text {Zeno }}$ as a function of $\tau$ is a decaying exponential. Its exponent has the dimensionality of an energy (in natural units); thus, it represents the global energy variation of $\mathcal{B}$ in the Zeno regime. In summary, the value of $\tau$ has to follow a trade-off condition: $\tau$ cannot be too small so as to prevent high-energy and entropic costs, but neither too large in order to avoid an inadequate value of the stabilization fidelity.

\section{CONCLUSION AND OUTLOOK}

Thermodynamics and control theory have been combined with the aim to stabilize an open quantum system that acts as a battery. In particular, in accordance with the laws of thermodynamics, we have analyzed the variation of the entropy generated on average by a quantum system, to be used as a device for the storage of energy, in interaction with an external environment and manipulated by a properly designed control action. In this respect, we have proposed a nonunitary control strategy, represented by a sequence of projective measurements interspersed by optimized time intervals, so that

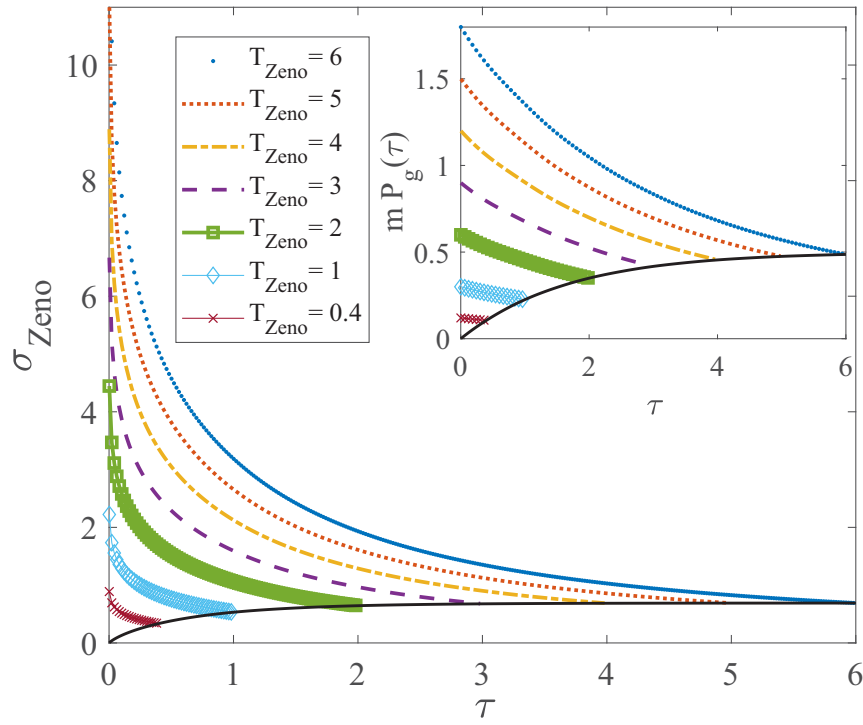

FIG. 3. Entropic cost of the Zeno protection procedure: $\sigma_{\text {Zeno }}$ as a function of the time interval $\tau$ between Zeno measurements. The results have been numerically derived for the same quantum system used in Fig. 2. Each curve has been obtained by choosing a fixed duration $T_{\text {Zeno }} \equiv t_{\text {fin }}-t_{\max }$, among a set of values (see the legend of the figure), and letting $\tau$ vary, so that also $\bar{m} \approx T_{\text {Zeno }} / \tau$ of Zeno measurements changes every time. The integral $\int_{0}^{\tau} \dot{H}(P) d t$ (black line), numerically solved with the initial condition $\rho=\rho_{e}$, has a monotonically increasing behavior for greater values of $\tau$, thus identifying $\bar{m}$ as the dominant factor. Inset: Amount of not stored energy $\bar{m} P_{g}(\tau)$ (normalized by $E_{\max }$ ) as a function of $\tau$ for $T_{\text {Zeno }}=0.4,1,2,3,4,5$, and 6 , in natural units. Here, the black line denotes $P_{g}(\tau)$, and an unavoidable worsening of the battery stabilization is observed when $\tau$ increases.

the control power is minimized. The introduced method can also be seen as a procedure to make the energy (Hamiltonian) basis of the system a decoherence-free subspace [53-55]. This implicitly corresponds to having engineered a superoperator $\mathcal{C}\left[\rho_{t}\right]$, modeling the average effects of applying projective measurements, so that for $\rho_{t} \approx \rho_{e}$,

$$
\dot{\rho}_{t}=-i\left[H_{0}, \rho_{t}\right]+\mathcal{D}\left[\rho_{t}\right]+\mathcal{C}\left[\rho_{t}\right]
$$

is approximately equal to zero at any time instant $t$.

As the main outlook, one could take into account the possibility that the projective measurements adopted in the stabilization scheme are nonideal, at least according to the definitions recently introduced in Ref. [52], so as to prevent an unbounded energy cost for their performance. Finally, other promising directions for future research lie in (i) the explicit treatment of charging and stabilization fluctuations, as was recently done for Gaussian quantum batteries [13], (ii) the adoption of optimal quantum control theory [22] to improve the stabilization procedure, (iii) the analysis for open quantum batteries with non-semigroup dynamics, e.g., non-Markovian behavior leading to memory effects, and (iv) the extension to multipartite quantum systems [9], exploiting the physics of quantum Zeno dynamics [55]. 


\section{ACKNOWLEDGMENTS}

This work was financially supported by the Fondazione CR Firenze through the project Q-BIOSCAN and QUANTUMAI, PATHOS EU H2020 FET-OPEN Grant No. 828946, and UNIFI Grant Q-CODYCES. F.C.B. acknowledges funding from the European Union's Horizon 2020 research and innovation programme under the Marie Skłodowska-Curie Grant Agreement No. 801110 and the Austrian Federal Ministry of Education, Science and Research (BMBWF).

\section{APPENDIX A: SECOND-LAW-LIKE INEQUALITY FOR STABILIZING AN OQB}

In this Appendix, we provide more details about the derivation of the minimum power required to stabilize an OQB affected by decoherence. Our proof follows a similar procedure to the analysis presented in Ref. [26].

Let us consider a quantum system dynamics described by the dynamical semigroup $\mathcal{V}(t) \equiv e^{\mathcal{L} t}$ with a (not necessarily unique) fixed point $\bar{\rho}_{\mathrm{dec}}=\mathcal{V}(t) \bar{\rho}_{\mathrm{dec}}$ and time-independent $\mathcal{L}$, where $\bar{\rho}_{\text {dec }}$ denotes the steady state induced by the presence of battery decoherence alone. As shown in Ref. [30], the corresponding entropy production rate $\Sigma\left(\rho_{t}\right)$ related to the battery density operator at time $t$ is convex and given by the following relation,

$$
\Sigma\left(\rho_{t}\right) \equiv-\operatorname{Tr}\left[\mathcal{L}\left[\rho_{t}\right]\left(\log \rho_{t}-\log \bar{\rho}_{\mathrm{dec}}\right)\right] \geqslant 0 .
$$

Recasting this general picture to the OQB model discussed in the main text, without applying external control for now, one has $\mathcal{L}\left[\rho_{t}\right]=D\left[\rho_{t}\right]$. Therefore, with our control knob given by a sequence of projective measurements, the entropy production rate $\Sigma\left(\rho_{t}\right)$ of the controlled OQB is greater than or equal to the entropy contribution $\Sigma_{D}\left(\rho_{t}\right)$ due to the environment alone. More formally, $\Sigma\left(\rho_{t}\right)=\Sigma_{D}\left(\rho_{t}\right)+\Sigma_{\mathrm{NU}}\left(\rho_{t}\right)$, with $\Sigma_{\mathrm{NU}}\left(\rho_{t}\right)$ denoting the entropy production rate of the battery given by the nonunitary control transformation. In other words, the total entropy production is lower bounded as

$$
\Sigma\left(\rho_{t}\right) \geqslant-\operatorname{Tr}\left[D\left[\rho_{t}\right]\left(\log \rho_{t}-\log \pi\right)\right] \equiv \Sigma_{D}\left(\rho_{t}\right) .
$$

Let us observe that by means of the control procedures the battery is stabilized in the sense that its density operator $\rho_{t}$ approaches the maximum energy state $\rho_{e}$, which thus becomes an equilibrium state induced by the control. Moreover, since we are assuming that any operation on $\mathcal{B}$ preserves the trace of its density operator, the total entropy production $\Sigma\left(\rho_{t}\right)$ is non-negative due to the monotonicity of relative entropies under CPTP maps. This means that only energy exchanges are allowed, such that the evolution of the uncontrolled battery can be always described by a CPTP quantum map.

As a next step, we quantify the rate of change of the battery total energy $E\left(\rho_{t}\right)$ under stabilizing control by using the first law of thermodynamics, with $\dot{E}\left(\rho_{t}\right)$ given by the relation

$$
\dot{E}\left(\rho_{t}\right)=\dot{E}_{D}\left(\rho_{t}\right)+\dot{W}_{\text {stab }}\left(\rho_{t}\right),
$$

where $\dot{E}_{D}\left(\rho_{t}\right)$ is the energy current driven into the battery by the environment, while $\dot{W}_{\text {stab }}\left(\rho_{t}\right)$ denotes the power required to charge $\mathcal{B}$ and stabilize it against decoherence. Here, the cost of the sequential measurements is included within the control cost $W_{\text {stab }}$. Our goal is to find a lower bound for $\dot{W}_{\text {stab }}\left(\rho_{t}\right)$. The energy $E_{D}\left(\rho_{t}\right)$ due to decoherence is given by

$$
E_{D}\left(\rho_{t}\right) \equiv \operatorname{Tr}\left[\rho_{t} H_{0}\right]-\operatorname{Tr}\left[\rho_{0} H_{0}\right],
$$

and the corresponding infinitesimal energy leakage is equal to

$$
\dot{E}_{D}\left(\rho_{t}\right)=\operatorname{Tr}\left[D\left[\rho_{t}\right] H_{0}\right] .
$$

If the battery is a two-level system, the energy current $\dot{E}_{D}\left(\rho_{t}\right)$ can be written as

$$
\dot{E}_{D}\left(\rho_{t}\right)=-T_{\bar{\rho}_{\mathrm{dec}}} \operatorname{Tr}\left[D\left[\rho_{t}\right] \log \bar{\rho}_{\mathrm{dec}}\right]=\dot{E}\left(\rho_{t}\right)-\dot{W}_{\mathrm{stab}}\left(\rho_{t}\right),
$$

where $T_{\bar{\rho}_{\text {dec }}}$ is the effective temperature of the battery in correspondence to the steady state $\bar{\rho}_{\mathrm{dec}}$. We point out that $T_{\bar{\rho}_{\mathrm{dec}}}$ is more than a parameter introduced for mathematical convenience: It corresponds to the physical temperature of a fictitious quantum system leading to the same decoherence effect as the general dynamics assumed here.

In this way, a lower bound for $\dot{W}_{\text {stab }}\left(\rho_{t}\right)$ can now be derived. We first reconsider Eq. (A1) again for the OQB in the absence of control,

$$
\Sigma_{D}\left(\rho_{t}\right)=-\operatorname{Tr}\left[D\left[\rho_{t}\right] \log \rho_{t}\right]+\operatorname{Tr}\left[D\left[\rho_{t}\right] \log \bar{\rho}_{\mathrm{dec}}\right] \geqslant 0 .
$$

Since $-\operatorname{Tr}\left[D\left[\rho_{t}\right] \log \rho_{t}\right]=\dot{S}_{D}\left(\rho_{t}\right)$ is the time derivative of the von Neumann entropy for the uncontrolled battery, we have that

$$
\dot{W}_{\text {stab }}\left(\rho_{t}\right)-\dot{E}\left(\rho_{t}\right) \geqslant-T_{\bar{\rho}_{\text {dec }}} \dot{S}_{D}\left(\rho_{t}\right),
$$

which leads to the analytical expression of the lower bound of $\dot{W}_{\text {stab }}$,

$$
\dot{W}_{\text {stab }}\left(\rho_{t}\right) \geqslant \dot{E}\left(\rho_{t}\right)-T_{\bar{\rho}_{\text {dec }}} \dot{S}_{D}\left(\rho_{t}\right) .
$$

Notice that Eq. (A9) has to fulfill the second law of thermodynamics. Indeed, by substituting $\dot{E}=\dot{E}_{D}+\dot{W}_{\text {stab }}$ from the first law of thermodynamics, the lower bound (A9) can be recast in the following inequality,

$$
\dot{F}\left(\rho_{t}\right) \equiv \dot{E}_{D}\left(\rho_{t}\right)-T_{\bar{\rho}_{\mathrm{dec}}} \dot{S}_{D}\left(\rho_{t}\right) \leqslant 0,
$$

where $F\left(\rho_{t}\right)$ stands for the free energy of the uncontrolled battery. Inequality (A10) implies that, without controlling the battery, its entropy unavoidably grows due to decoherence, leading thus to a progressive decreasing of the battery free energy.

Here, it is worth observing that the lower bound (A9) is quite conservative, in the sense that the value provided for the minimum control power $\dot{W}_{\text {stab }}$ could be overestimated. This is because we have not directly expressed $\dot{W}_{\text {stab }}$ as a function of the entropy production rate $\Sigma_{\mathrm{NU}}\left(\rho_{t}\right)$ given by controlling $\mathcal{B}$ by means of the proposed nonunitary transformation (sequence of projective measurements). $\Sigma_{\mathrm{NU}}\left(\rho_{t}\right)$ is equal to the sum of the entropy production rates associated with each projective measurement, and it can be obtained by evaluating the energy cost in storing and erasing the measurement outcomes in relation to Landauer's principle [48-50]. In this regard, by considering the expression for the entropic contribution $s_{t}$ of each single measurement result, i.e., $s_{t} \equiv-\log P$, with $P$ (equal to $P_{e}$ or $P_{g}$ ) denoting the probability that the battery collapses in one of the two energy eigenstates [47], we find that $\Sigma_{\mathrm{NU}}\left(\rho_{t}\right)=$ 
$\dot{H}(P)=-\dot{P}_{e} \log \left(\frac{P_{e}}{1-P_{e}}\right)$, where $\dot{H}$ denotes the time derivative of the Shannon entropy $H(P) \equiv-\sum_{k \in\{e, g\}} P_{k} \log P_{k}$, with $P_{g}=1-P_{e}$. It is worth noting that the entropy production $\Sigma_{\mathrm{NU}}$ is zero if and only if $P_{g}=P_{e}=1 / 2$, i.e., the probabilities that the battery collapses in the maximum or lowest-energy state are both equal to $1 / 2$.

\section{APPENDIX B: DETAILS ABOUT THE NUMERICAL IMPLEMENTATION}

The results of Fig. 2 in the main text have been obtained by considering as a quantum battery the following two-level system, with an internal Hamiltonian $H_{0}=\Omega \sigma_{x}+\omega \sigma_{z}$, where $\omega=1, \Omega=3$ (in units such that $\hbar=1$ ), and $\sigma_{x}, \sigma_{z}$ Pauli matrices. Thus, in the basis of $\sigma_{z}$, given for convention by the eigenstates $|0\rangle \equiv[0,1]^{T}$ and $|1\rangle \equiv[1,0]^{T}$ [the superscript $(\cdot)^{T}$ denotes the transposition symbol], the corresponding maximum and minimum energy states are respectively equal to

$$
\rho_{e} \approx\left(\begin{array}{ll}
0.658 & 0.474 \\
0.474 & 0.342
\end{array}\right) \text { and } \rho_{g} \approx\left(\begin{array}{cc}
0.342 & -0.474 \\
-0.474 & 0.658
\end{array}\right) .
$$

Here, we have reasonably chosen as the input density operator $\rho_{0}$ the minimum energy state $\rho_{g}$. Moreover, in order to fulfill the fast control condition, we have assumed to use a time-dependent term $V(t)$ in the driving Hamiltonian only to slightly bring out of equilibrium the battery from $\rho_{g}$ to $\equiv|0\rangle\langle 0|$. Given the internal time-independent Hamiltonian $H_{0}$, this operation is achieved by taking $V(t)=e^{-i \phi \sigma_{y}}$ (rotation around the $y$ axis), with

$$
\phi \equiv \arctan \left(\frac{\rho_{g}^{(11)}}{\rho_{g}^{(21)}}\right) .
$$

Then, to transfer the battery population from $|0\rangle\langle 0|$ to the initialization state $\rho_{i}$, only the dynamical evolution governed by $H_{0}$ has been exploited. Accordingly, under this assumption, we need to determine the optimal value of $t$ (i.e., $\left.t^{*}\right)$ in correspondence to which it is worth performing the first energy projective measurement of the protocol.

The dynamical evolution of the system is given by the Markovian master equation $\dot{\rho}_{t}=-i\left[H_{0}, \rho_{t}\right]+D\left[\rho_{t}\right](\hbar=1)$, where the superoperator $D\left[\rho_{t}\right]$ modeling decoherence within the battery dynamics has been chosen equal to $D\left[\rho_{t}\right]=$ $\gamma\left(-\left\{\mathcal{N}, \rho_{t}\right\}+2 \mathcal{N} \rho_{t} \mathcal{N}\right)$, i.e., as an operator inducing pure dephasing, with $\{\cdot, \cdot\}$ the Poisson bracket, $\mathcal{N} \equiv|1\rangle\langle 1|$, and $\gamma=2 / 3$. Here, it is worth noting that also pure-dephasing master equations, despite being energy preserving, involve dynamical behaviors worthy of being studied, since stabilizing an OQB implicitly implies the protection (in our case) of coherence in the battery energy basis. Moreover, the motivation under the choice of $\gamma=2 / 3$ will be clear below. In Fig. 4 we show the behavior over time of both the populations and coherence of the two-level system for a whole duration of the dynamics taken equal to 10 (always in natural units) by starting from the state $|0\rangle\langle 0|$. Instead, in Fig. 5 we plot the trace distance of $\rho_{t}$ with respect to $\rho_{e}$, as well as the probability $P_{e}(t) \equiv \operatorname{Tr}\left[\rho_{t} \rho_{e}\right]$. We can observe that the trace distance $T\left(\rho_{t}, \rho_{e}\right)$ is always greater than or equal to $1 / 2$, (a)

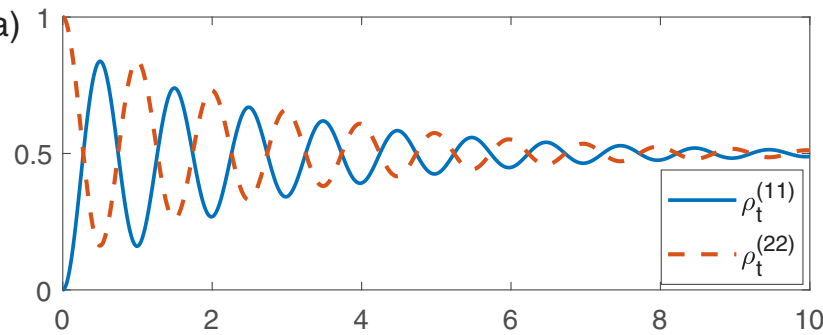

(b)

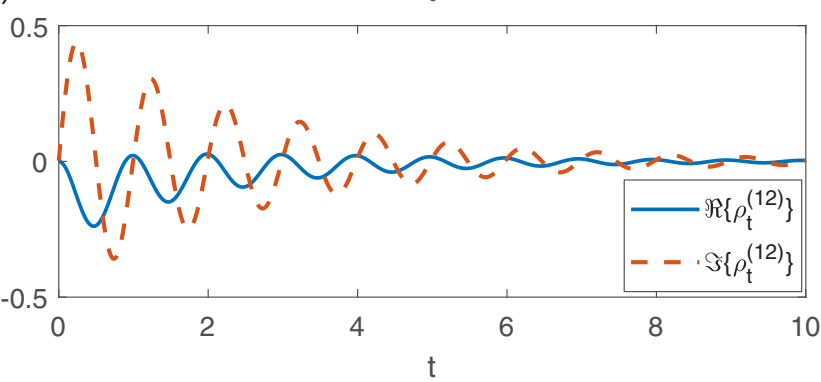

FIG. 4. (a) Behavior over time of the battery density matrix elements $\rho_{t}^{(11)}$ and $\rho_{t}^{(22)}=1-\rho_{t}^{(11)}$. (b) Behavior over time of the real and imaginary part of the battery density matrix element $\rho_{t}^{(12)}$.

so that $P_{g} \geqslant 1 / 2$. In this regard, in the numerical simulations we have verified that also in this case $\left\langle\Delta E_{\text {meas }}(t)\right\rangle \equiv$ $P_{g} \operatorname{Tr}\left[H_{0}\left(\rho_{t}-\rho_{g}\right)\right]+P_{e}\left[H_{0}\left(\rho_{e}-\rho_{t}\right)\right] \approx 0$ for each $t$ within the entire evolution of the system, and thus also for $t^{*}$.

The results in Figs. 4 and 5 could induce the experimenter to take as $t^{*}$ a sufficiently long time interval so as to minimize the trace distance $T\left(\rho_{t}, \rho_{e}\right)$ and at the same time maximize the probability $P_{e}$. However, because the stabilization scheme is a probabilistic procedure, this choice could bring the main disadvantage of waiting for a long time interval and then

(a)
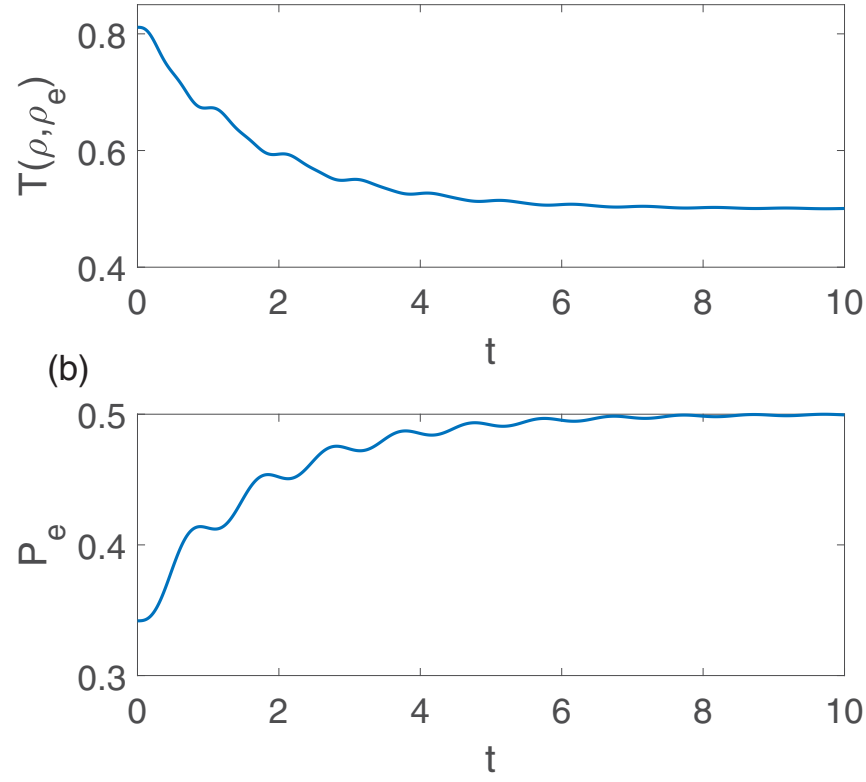

FIG. 5. (a) Behavior over time of the trace distance $T\left(\rho_{t}, \rho_{e}\right)$. (b) Behavior over time of the probability $P_{e}$. 
observing the battery staying for most of the time not in the maximum energy state but in correspondence to $\rho_{g}$, and thus leading to a very low stabilization fidelity $\mathcal{F}$. To make a better choice of $t^{*}$, it is worth analyzing the reason why $P_{e}$ is always $\leqslant 1 / 2$. We find that, in order to achieve the maximum energy state $\rho_{e}$, we need to stabilize both populations and coherence of the battery, but with the chosen internal Hamiltonian $H_{0}$ the stabilization of populations and coherence cannot be reached at the same time. In this regard, there are three possibilities: (a) Minimize only the difference between the modulus squared of coherence terms of $\rho_{t}$ and $\rho_{e}$; (b) minimize only the difference between the modulus squared of the diagonal terms of $\rho_{t}$ and $\rho_{e}$; and (c) find a trade-off between (a) and (b) by ensuring that the value of $t^{*}$ is not too large and at the same time $\left\langle W_{\text {stab }}\right\rangle$ is as small as possible with respect to the average energy leakages $\langle\Delta L\rangle$. For the specific implementation of Fig. 2 in the main text we have chosen solution (c) corresponding to a value of $t^{*}$ equal to 0.33 (in natural units). Notice that, since $P_{e} \leqslant 1 / 2$ by starting from the state $|0\rangle\langle 0|$, we can at most minimize the average total control work and get a very high fidelity $\mathcal{F}$, but without achieving the best possible energetic efficiency. We have deliberately chosen this example in order to show that the proposed stabilization scheme, based on sequential quantum measurements, turns out to be extremely efficient from an energetic point of view only if the probability to collapse onto $\rho_{e}$ after each quantum measurement is sufficiently high, ideally close to 1 . If not, a greater energy cost (if compared with $\left.E_{\max }\right)$ is required, so as to bring the system into the maximum energy state and at the same time compensate the presence of the external environment leading to decoherence.

Finally, as it can be observed in Fig. 2 in the main text, the probability that the state of the battery collapses in the minimum energy state $\rho_{g}$ while the procedure of Zeno protection is turned on is very low (smaller than $1 \%$ ). This is due to our choice to take the time interval $\tau$ between Zeno measurements to be equal to 0.0662 (in natural units), five times smaller than $t^{*}$. However, there does exist the possibility that the Zeno protection procedure would fail; in such a case we simply reinitialize the system and the stabilization scheme is repeated from the beginning. In this regard, it is worth noting that for larger values of $\tau$ the fidelity $\mathcal{F}$ decreases and consequently $\left\langle W_{\text {stab }}\right\rangle$ unavoidably increases, since for a fixed value of $T_{\text {Zeno }}$ the stabilization scheme needs to be reinitialized a greater number of times.
[1] R. Kosloff, Entropy 15, 2100 (2013).

[2] J. Goold, M. Huber, A. Riera, L. del Rio, and P. Skrzypczyk, J. Phys. A: Math. Theor. 49, 143001 (2016).

[3] S. Vinjanampathy and J. Anders, Contemp. Phys. 57, 545 (2016).

[4] Thermodynamics in the Quantum Regime, edited by F. Binder, L. A. Correa, C. Gogolin, J. Anders, and G. Adesso (Springer, Berlin, 2018).

[5] S. Deffner and S. Campbell, Quantum Thermodynamics (Morgan \& Claypool Publishers, San Rafael, CA, 2019).

[6] F. Campaioli, F. A. Pollock, and S. Vinjanampathy, in Thermodynamics in the Quantum Regime, edited by F. Binder, L. Correa, C. Gogolin, J. Anders, G. Adesso, Fundamental Theories of Physics Vol. 195 (Springer, Cham, 2018), Chap. 8, pp. 207-225.

[7] R. Alicki and M. Fannes, Phys. Rev. E 87, 042123 (2013).

[8] P. Skrzypczyk, A. A. J. Short, and S. Popescu, Nat. Commun. 5, 4185 (2014).

[9] F. C. Binder, S. Vinjanampathy, K. Modi, and J. Goold, New J. Phys. 17, 075015 (2015).

[10] F. C. Binder, Work, heat, and power of quantum processes, D.Phil. thesis, University of Oxford, 2016.

[11] F. Campaioli, F. A. Pollock, F. C. Binder, L. Céleri, J. Goold, S. Vinjanampathy, and K. Modi, Phys. Rev. Lett. 118, 150601 (2017).

[12] D. Ferraro, M. Campisi, G. M. Andolina, V. Pellegrini, and M. Polini, Phys. Rev. Lett. 120, 117702 (2018).

[13] N. Friis and M. Huber, Quantum 2, 61 (2018).

[14] G. M. Andolina, D. Farina, A. Mari, V. Pellegrini, V. Giovannetti, and M. Polini, Phys. Rev. B 98, 205423 (2018).

[15] D. Farina, G. M. Andolina, A. Mari, M. Polini, and V. Giovannetti, Phys. Rev. B 99, 035421 (2019).

[16] G. M. Andolina, M. Keck, A. Mari, M. Campisi, V. Giovannetti, and M. Polini, Phys. Rev. Lett. 122, 047702 (2019).
[17] S. Julià-Farré, T. Salamon, A. Riera, M. N. Bera, and M. Lewenstein, arXiv:1811.04005.

[18] F. Barra, Phys. Rev. Lett. 122, 210601 (2019).

[19] L. P. Garcia-Pintos, A. Hamma, and A. del Campo, arXiv:1909.03558.

[20] A. C. Santos, B. Çakmak, S. Campbell, and N. T. Zinner, Phys. Rev. E 100, 032107 (2019).

[21] J. Liu, D. Segal, and G. Hanna, J. Phys. Chem. C 123, 18303 (2019).

[22] C. Brif, R. Chakrabarti, and H. Rabitz, New J. Phys. 12, 075008 (2010).

[23] C. P. Koch, J. Phys.: Condens. Matter 28, 213001 (2016).

[24] J. M. Horowitz and J. L. England, Entropy 19, 333 (2017).

[25] F. Ticozzi, K. Nishio, and C. Alta, IEEE Trans. Autom. Control 58, 74 (2013).

[26] J. M. Horowitz and K. Jacobs, Phys. Rev. Lett. 115, 130501 (2015).

[27] J. J. W. H. Sørensen, M. Dalgaard, A. H. Kiilerich, K. Mølmer, and J. F. Sherson, Phys. Rev. A 98, 062317 (2018).

[28] R. Dann, A. Tobalina, and R. Kosloff, Phys. Rev. Lett. 122, 250402 (2019).

[29] H.-P. Breuer and F. Petruccione, The Theory of Open Quantum Systems (Oxford University Press, Oxford, UK, 2002).

[30] H. Spohn, J. Math. Phys. 19, 1227 (1978).

[31] P. A. Camati, J. P. S. Peterson, T. B. Batalhão, K. Micadei, A. M. Souza, R. S. Sarthour, I. S. Oliveira, and R. M. Serra, Phys. Rev. Lett. 117, 240502 (2016).

[32] S. Gherardini, M. M. Müller, A. Trombettoni, S. Ruffo, and F. Caruso, Quantum Sci. Technol. 3, 035013 (2018).

[33] T. Batalhão, S. Gherardini, J. Santos, G. Landi, and M. Paternostro, in Characterizing Irreversibility in Open Quantum Systems, edited by F. Binder, L. Correa, C. Gogolin, J. Anders, G. Adesso, Thermodynamics in the Quantum Regime, Vol. 195 (Springer, Cham, 2018), Chap. 16, pp. 395-410. 
[34] A. De Pasquale, K. Yuasa, and V. Giovannetti, Phys. Rev. A 96, 012316 (2017).

[35] S. Gherardini, A. Smirne, M. M. Müller, and F. Caruso, Proceedings 12, 11 (2019).

[36] M. Campisi, P. Talkner, and P. Hänggi, Phys. Rev. Lett. 105, 140601 (2010).

[37] F. W. J. Hekking and J. P. Pekola, Phys. Rev. Lett. 111, 093602 (2013).

[38] S. Gherardini, L. Buffoni, M. M. Müller, F. Caruso, M. Campisi, A. Trombettoni, and S. Ruffo, Phys. Rev. E 98, 032108 (2018).

[39] C. Elouard, D. Herrera-Martí, B. Huard, and A. Auffèves, Phys. Rev. Lett. 118, 260603 (2017).

[40] L. Buffoni, A. Solfanelli, P. Verrucchi, A. Cuccoli, and M. Campisi, Phys. Rev. Lett. 122, 070603 (2019).

[41] A. E. Allahverdyan, R. Balian, and T. M. Nieuwenhuizen, Europhys. Lett. 67, 565 (2004).

[42] N. Suri, F. C. Binder, B. Muralidharan, and S. Vinjanampathy, Eur. Phys. J.: Spec. Top. 227, 203 (2018).

[43] A. Smerzi, Phys. Rev. Lett. 109, 150410 (2012).

[44] F. Schäfer, I. Herrera, S. Cherukattil, C. Lovecchio, F. S. Cataliotti, F. Caruso, and A. Smerzi, Nat. Commun. 5, 3194 (2014).
[45] M. M. Müller, S. Gherardini, A. Smerzi, and F. Caruso, Phys. Rev. A 94, 042322 (2016).

[46] W. K. Wootters, Phys. Rev. D 23, 357 (1981).

[47] P. Strasberg, Phys. Rev. E 100, 022127 (2019).

[48] S. Lorenzo, R. McCloskey, F. Ciccarello, M. Paternostro, and G. M. Palma, Phys. Rev. Lett. 115, 120403 (2015).

[49] L. Mancino, M. Sbroscia, E. Roccia, I. Gianani, F. Somma, P. Mataloni, M. Paternostro, and M. Barbieri, npj Quantum Inf. 4, 20 (2018).

[50] K. Abdelkhalek, Y. Nakata, and D. Reeb, arXiv:1609.06981.

[51] For a fixed value both of $\tau$ and $t_{\text {fin }}$ also the number of Zeno measurements is a random variable, since an unsuccessful projection on $\rho_{g}$ can happen, in spite of a very small occurrence probability for such phenomenon.

[52] Y. Guryanova, N. Friis, and M. Huber, Quantum 4, 222 (2020).

[53] D. A. Lidar and B. K. Whaley, in Irreversible Quantum Dynamics, edited by F. Benatti and R. Floreanini (Springer, Berlin, 2003), pp. 83-120.

[54] P. G. Kwiat, A. J. Berglund, J. B. Altepeter, and A. G. White, Science 290, 498 (2000).

[55] M. M. Müller, S. Gherardini, and F. Caruso, Ann. Phys. 529, 1600206 (2017). 